\title{
Effectiveness analysis of active space debris removal technologies for the geostationary orbit.
}

\author{
A.A. Vnukov \\ Research and Production Center Small Sattelites, LLC \\ Russian Federation, 662971, Krasnoyarsk krai, \\ Zheleznogorsk, Shkolnaya st., 33-25
}

\author{
T.N. Balandina \\ JSC Academician M.F. Reshetnev Information Satellite \\ Systems \\ Russian Federation, 662972, Krasnoyarsk krai, \\ Zheleznogorsk, Lenina st., 52 \\ E-mail:vnukovalx@ya.ru
}

\begin{abstract}
This paper considers the basic technologies of active removal of space debris from the geostationary orbit. For two of them: coupling of service spacecraft (SC) with the space debris item with the help of an arm of the service SC (rigid link) and capture of the space debris object with a net or a harpoon (flexible link), - a list of main disturbing moments is given, influencing the service SC during the coupling and space debris towing to the graveyard orbit. A comparative analysis of resource intensity has been performed of various schemes for space debris removal with rigid or flexible link.
\end{abstract}

Key words: space debris, geostationary orbit, external removal of space debris, rigid link, flexible link, disturbing moments.

\section{INTRODUCTION}

The problem of cleaning the GEO (geosynchronous equatorial orbit) from space debris lays in its being impossible or impractical to passively remove non-functioning SCs and last stages of launch vehicles from the protected area of the GEO. Due to low eccentricity of SC (spacecraft) in GEO, natural evolution of pericenter and apogee altitudes is so small, that despite periodic (with a period of about 15 years) fluctuations of these values, the average pericenter altitude, for example, changes by $10 \mathrm{~km}$ maximum in 100 years. It is an unacceptably low value from the point of view of requirements of national and international standards with respect to avoidance of collision between SDOs (space debris object) and operating SC in GEO [1].

Due to that, various aerospace agencies conduct research on possibility of active interaction with the SDOs in GEO with the aim to move them into a graveyard orbit. Three concepts are the most developed:

- SSC (service spacecraft) approaching an SDO to a distance of several meters, rigid capture of the SDO with an arm installed on the SSC, orientation of the SDO-SSC pair with the line of action of SSC's main propulsion along the vector of orbital velocity and then controlled towing of the SDO-SSC cluster to a graveyard orbit;

- SSC approaching an SDO to a distance of tens of meters, remote capture of the SDO with a harpoon or a net thrown from the SSC, orientation of the SSC with the line of action of SSC's main propulsion along the vector of orbital velocity and then towing the SDO to a graveyard orbit;
- SSC approaching an SDO to a distance of several dozens of meters, action in a direction of the SDO's center of mass along the vector of orbital velocity of the SDO-SSC cluster with a reactive jet, thus creating a necessary impulse to drive the SDO to a graveyard orbit.

This paper gives a comparative efficiency analysis of mechanical means of action on SDO as they are the most welldeveloped and have relatively simple implementation path.

\section{OVERVIEW OF ORBITAL COUPLING PROJECT WITH THE USE OF AN ARM}

Developers of orbital coupling between the SSC and SDO with the help of arms have achieved the most viable results. First of all it is due to the fact that the arms were actively employed in the Space Shuttle program and are currently in use on the International Space Station.

An arm is a multisegment mechanism equipped with a clutch or a camera and sometimes with both of them. Rotation of each segment is provided by electric motors integrated with joints by design. Sufficient number of links allow providing necessary universality of the arm, but makes controlling the arm, as well as SSC where it is installed a more complex task.

An arm developed in the DEOS project is shown in Figure 1. Its weight is $40 \mathrm{~kg}$, of which about $36 \mathrm{~kg}$ is the weight of links and joints and $4 \mathrm{~kg}$ is the weight of the clutch [2].

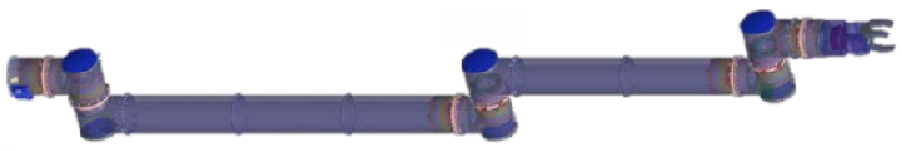

Fig. 1. Project DEOS arm

Within the framework of Project Phoenix, an arm known as FREND has been developed, with a weight of $78 \mathrm{~kg}$, plus additional $10 \mathrm{~kg}$ is the weight of electronic control module [3]. 


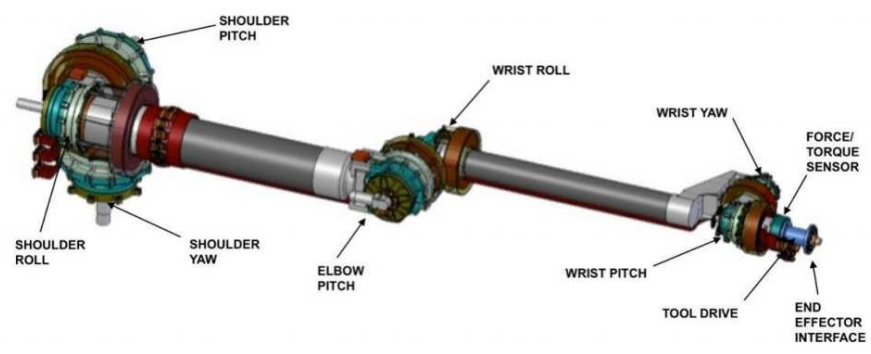

Fig. 2. FREND arm from Project Phoenix

Tethers Unlimited has developed a prototype of KRAKEN arm [4], which is a small and light structure developed with the aim of being employed in mini- and micro-scale satellites that perform tasks of orbital assembly, SC maintenance and cleaning orbits from SDOs. Design of the arm allows collapsing it to a volume of one and a half liter, at that KRAKEN is able to perform operations in the hemisphere of a one-meter radius. Thanks to its modular design, KRAKEN's outreach hemisphere may be extended to a two-meter radius by adding new links into the arm design.

General layout of the KRAKEN arm with seven degrees of freedom is shown in Figure 3, main features of the system are given in Table 1.

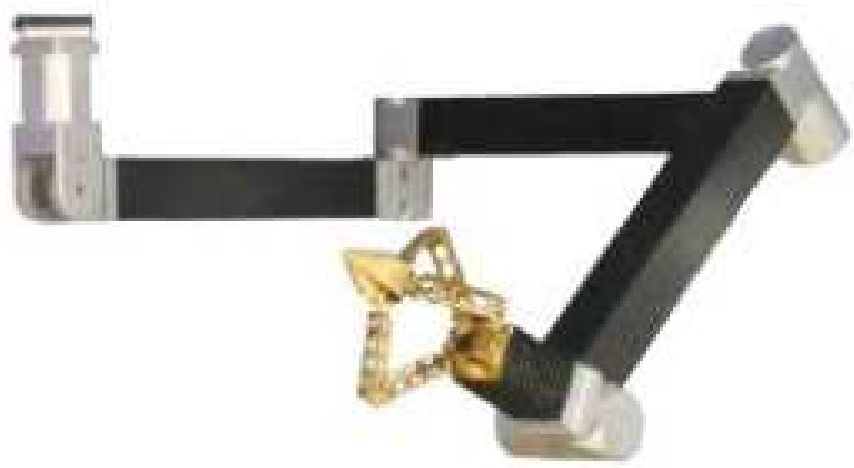

Fig. 3. Layout of KRAKEN collapsible arm

TABLE I. MAIN FEATURES OF KRAKEN ARM

\begin{tabular}{|l|c|}
\hline \multicolumn{1}{|c|}{ Parameter } & Value \\
\hline Arm length, $\mathrm{m}$ & $\leq 2$ \\
\hline Degrees of freedom & $\leq 11$ \\
\hline Volume in collapsed state, 1 & 1.5 \\
\hline Positioning precision, $\mathrm{mm}$ & \pm 10 \\
\hline Total weight of the system, $\mathrm{kg}$ & 4.2 \\
\hline
\end{tabular}

\section{Overview of orbital coupling project using flexible}

link

Flexible links are less well-studied due to their one-off nature: a mistake in aiming and capture of a test SDO may lead to loss of contacting part of the flexible link (net or harpoon) or appearance one more SDO in orbit, that is why field test of flexible link method has not been performed yet and all the studies are performed exclusively on digital models.

ROGER (RObotic GEostationary orbit Restorer) concept [5] was suggested by European Space Agency in 2014. Service SC with the mass of $3500 \mathrm{~kg}$ can inspect serviced SC, stabilize it or move to different orbits using a capture system consisting of a net thrown to a distance that excludes possibility of collision between the service SC and the SC being serviced (Figure 4). Primarily, this multi-use system was intended for deorbiting non-cooperative SCs from target orbits to graveyard orbits.

It was expected, that a service spacecraft will be developed on the basis of satellite backbone previously developed in Airbus. Some insignificant variation in design of SCA for different missions is possible.

The servicing SC is provided with an apogee motor (fuel weight $-2700 \mathrm{~kg}$, may be extended to 3200 кг, 22 liquid propellant systems with $10 \mathrm{~N}$ of motive power and an apogee motor with a motive power of $400 \mathrm{~N}$ ), the number of engines in which may vary depending on the aim of the mission. Solar panels serve as the source of electric power. Internal energy usage of the $\mathrm{SC}$ is $300 \mathrm{~W}$.
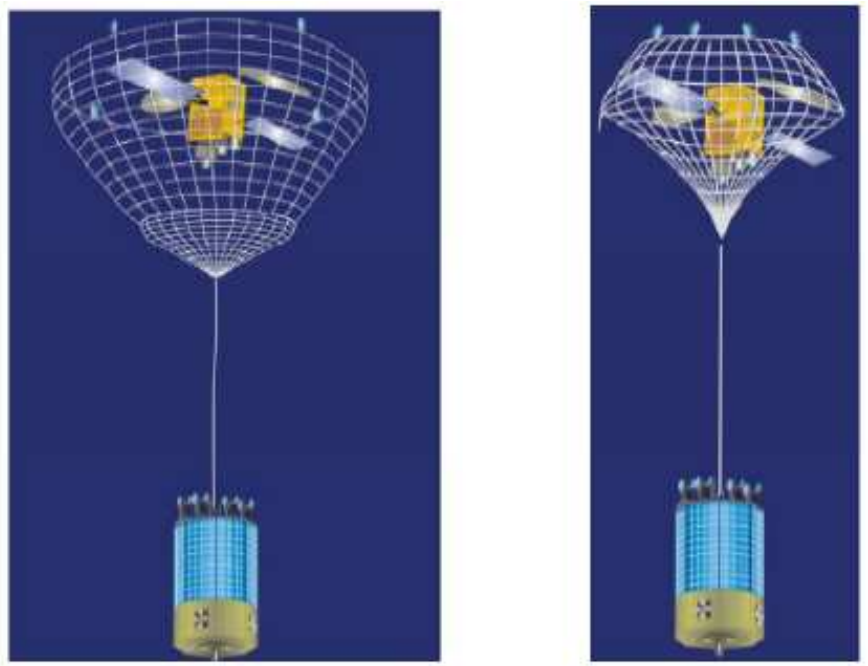

Fig. 4. ROGER concept

Main difficulties in developing the system:

- ability of safe capture of a target satellite and changing its dynamics, variability of forms and dimensions of target SCs, rigid surfaces and possible coupling units (accuracy of SC aiming is less than $0.25^{\circ}$ );

- $\quad$ optimization of the number of target SCs that may be deorbited to a graveyard orbit during a single mission of the mother SC taking into account refueling and time spent deorbiting the target $\mathrm{SC}$ to the graveyard orbit;

- possibility to create mobile robotic capture systems (assumed weight of the mechanism is $9 \mathrm{~kg}$, size of the net is $10 \times 10 / 15 \times 15 \mathrm{~m}$, transport volume is $5 \mathrm{l}$ ). 
There is also a steel net for collecting space debris developed by Japan Aerospace Exploration Agency (JAXA) (Figure 5) [6].

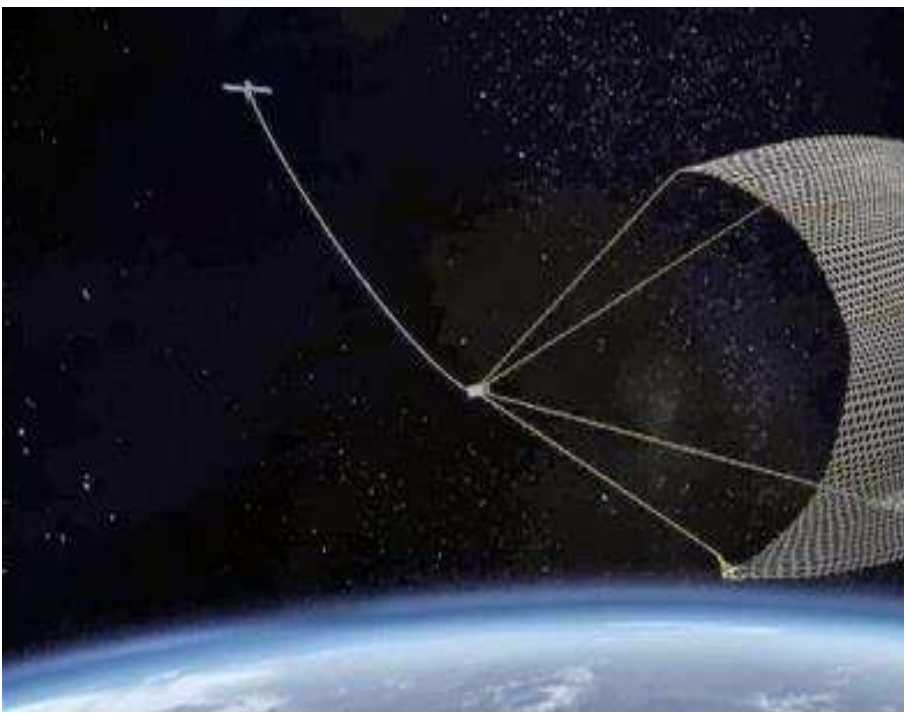

Fig. 5. Figure $5-$ Steel net for collection of space debris

In 2016, scientists from the Japanese space agency attempted testing an electrodynamic "leash" created with participation of a fishnet manufacturer to slow an orbiting fragment of space debris and deorbiting it to a lower orbit from where it shall further ingress into dense atmosphere and burn safely.

A 700-meter mesh made of fine stainless aluminum-steel wire had to be released from the board of a spacecraft having fulfilled its mission to deliver goods to ISS and decoupled for further burning in the Earth's atmosphere, but the leash was never released. For a week Japanese specialists attempted various solutions to no avail.

A harpoon developed by Airbus Defence \& Space (formerly Astrium) [7], is considered the most appropriate technology for space debris capture among all the technologies studied by EADS in recent years. Its advantages are: compatibility with various types of space debris (terminal rockets or spacecrafts); simplicity of technology ground work and relative tolerance for rotation of the SDO or location of anchor point. The system consists of the harpoon itself, a launching unit and a rope. The harpoon consists of a barbed tip providing engagement with the SDO, a crushable cartridge for controlling the penetration depth, a shaft providing interaction with the launcher and a stabilizer for ground-based testing. The launcher runs on compressed nitrogen and may be used to launch several harpoon installed on the SSC. After taking aim and launching the harpoon, the SSC stays linked to the SDO by means of the harpoon and Dyneema rope, stored in the SSC coiled on a spool. The rope is designed for a maximum load of $1.6 \mathrm{kN}$.

General layout of the harpoon is shown in Figure 6, main features of the system are given in Table 3 .

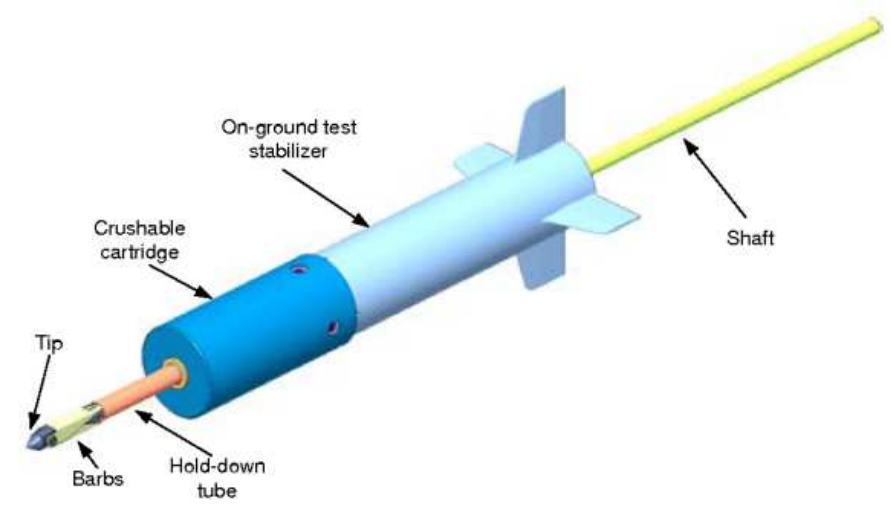

Fig. 6. Layout of EADS harpoon

TABLE II. MAIN FEATURES OF ASTRIUM HARPOON

\begin{tabular}{|l|l|}
\hline \multicolumn{1}{|c|}{ Parameter } & \multicolumn{1}{c|}{ Value } \\
\hline SDO mass, kg & $\leq 9000$ \\
\hline System dimensions, mm & $585 \times 400$ \\
\hline Power consumption, W & 20 \\
\hline Shooting distance, $\mathrm{m}$ & $\leq 10$ \\
\hline Harpoon weight, kg a system with two & 1.3 \\
\hline $\begin{array}{l}\text { Total mass of a } \\
\text { harpoons, kg }\end{array}$ & 8 \\
\hline
\end{tabular}

\section{Disturbing MOMENTS IN COUPLING THE SSC WITH} AN SDO

When organizing a mechanical link with an SDO performing a motion with respect to the SSC center of mass, a kinetic moment appears, quantitatively equal to the product of the moment of inertia of the body with respect to its rotational axis and its angular velocity [8]:

$$
\left\{\begin{array}{l}
K_{X}=J_{X} \cdot \omega_{X} \\
K_{Y}=J_{Y} \cdot \omega_{Y} \\
K_{Z}=J_{Z} \cdot \omega_{Z}
\end{array}\right.
$$

In this case, the rotational axis is assumed to be the attachment point of the coupling system (an arm or a flexible link) to the SSC.

To provide controllable movement of the SDO-SSC tandem, the orientation and stabilization system shall create a controlling moment equal to the time derivative of the SSC's kinetic moment caused by the coupling with the SDO:

$$
\left\{\begin{array}{l}
M_{X}=\frac{d K_{X}}{d t}=\frac{d\left(J_{X} \cdot \omega_{X}\right)}{d t} \\
M_{Y}=\frac{d K_{Y}}{d t}=\frac{d\left(J_{Y} \cdot \omega_{Y}\right)}{d t} \\
M_{Z}=\frac{d K_{Z}}{d t}=\frac{d\left(J_{Z} \cdot \omega_{Z}\right)}{d t}
\end{array}\right.
$$

Taking SDO moment of inertia as constant and assuming change to the SSC angular velocity due to coupling with the 
SDO as being momentary, we may determine, that the value of the controlling moment depends exclusively on the SDO angular velocity with respect to the SSC.

Taking into account, that $\omega=\frac{V}{R}$, where $V$ is a projection of SDO orbital velocity onto a corresponding SSC coordinate axis, and $R$ is a distance between the SSC and SDO, the value of necessary compensating moment is inversely proportional to the length of the mechanical link between the SSC and the SDO.

By definition, flexible link is used to capture SDOs at a distance which is several dozens of meters longer than the length of SSC arms, thus, to compensate the disturbing moments from a flexible link would be tens of times more difficult on behalf of the SSC than to parry any disturbing moments coming from an arm.

However, besides the kinetic moment arising due to motion of the SDO with respect to the SSC, the arm creates kinetic moment that changes SSC orientation in space during rotation of its segments around its joints. That is is why controlling moments shall be created to compensate the disturbing moments from arm operations, e.g., with flywheels. For instance, for an SSC provided with a two-link arm (Figure 7), an attempt to move the arm's clutch from point A to point B will lead to change in position of the SSC in space as shown in Figure 8 [9].

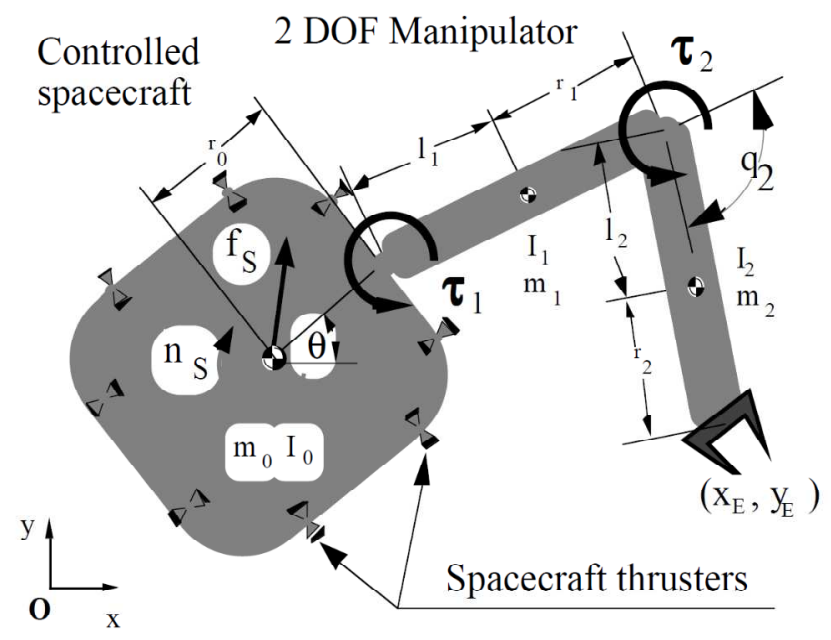

Fig. 7. SSC with a two-segment arm

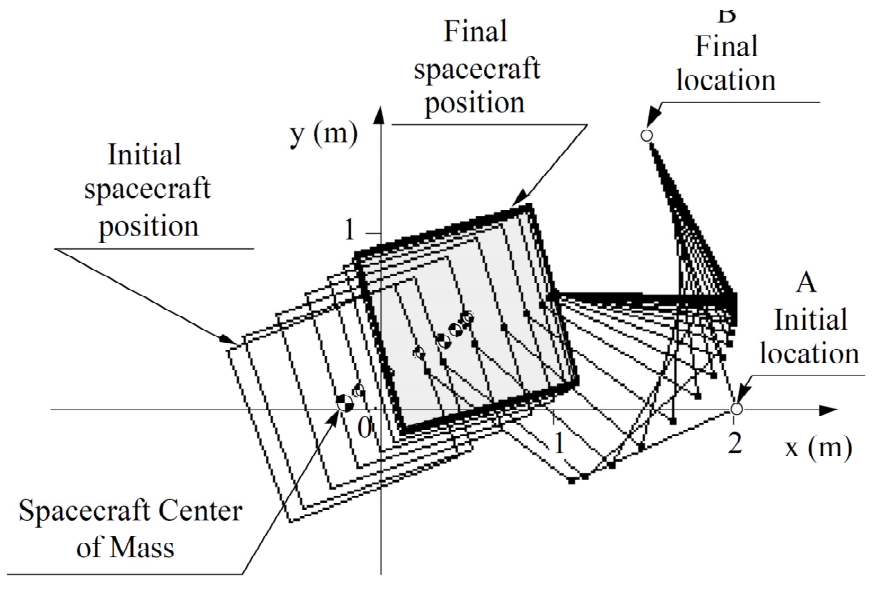

Fig. 8. Operational kinematics of a two-link arm of a SSC

Thus, SSC resource costs in coupling with an SDO using an arm are a combination of costs to parry the disturbing kinetic moment from SDO motion with respect to the SSC and costs to parry the disturbing kinetic moments caused by movement of separate segments of the arm with respect to the SSC. However, as the weight of the segments is small compared to the weight of the SDO, we may neglect the costs of parrying the kinetic moment from movement of arm segments.

\section{COMPARATIVE EFFICIENCY ANALYSIS OF SC WITH ARMS AND SC WITH A FLEXIBLE LINK}

Coupling with an arm is currently the most well-developed SSC-SDO coupling method. This method has an extensive operating experience and has been tested in orbit in automatic mode many times. The coupling system employing a flexible link still has not been tested in flight.

The weight of the arm may be from ten to forty kilogram, depending on segment length. Efficient coupling would require two arms minimum: one for coupling and the second one to carry optical coupling control means. Thus, total weight of the arm-enabled coupling system would approach $100 \mathrm{~kg}$.

Weight of the flexible link (a mesh or a harpoon) is under $10 \mathrm{~kg}$. Efficient operation of the flexible-link-enabled coupling system does not require external video surveillance and cameras may be placed on the SSC body.

Disturbing moments caused by SDO movement with respect to the SSC are tens of times higher for a flexible-linkenabled system than for an arm-enabled, thus, SSC costs to parry them would be tens times higher as well. However, if the parrying is performed by electromechanical means (flywheels and gyroscopes), the resource spent for parrying is renewable, thus mitigating the difference. It is possible when the weight of the SDO is less than or comparable with the weight of the SSC.

The arm is a reusable system and after finishing the maneuver to put the SDO into a graveyard orbit, it may be used again, while the flexible link foresees SSC decoupling from the SDO only by means of disconnecting the flexible system on the SSC side, thus reuse of a flexible-link-enabled 
SC would require providing it with several flexible link systems. Thus, the flexible link's mass efficiency is meaningful only if the SSC is intended for removal of a single SDO.

\section{CONCLUSIONS}

SSCs equipped with arms are efficient for repeated removal of SDO with a weight larger that that of the SSC itself, at that, to parry the disturbing kinetic moments coming from a large SDO, non-flow systems may be employed (electromechanical actuator mechanisms), as well as systems using a non-renewable resources (jet engines).

SSCs equipped with flexible link are efficient for a one-off removal of SDO with a weight lower than that of the SSC, at that, it is practical to use only non-flow systems to compensate the disturbing kinetic moments arising.

\section{Acknowledgment}

The work has been carried out in the framework of applied research on the topic "Development of the concept of a service spacecraft for cleaning the region of geosynchronous equatorial orbit (GEO) of space debris of technogenic nature" with the financial support of the Ministry of Education and Science of the Russian Federation; the unique identifier of the agreement is RFMEFI57617X00932.

\section{References}

1. GOST R 52925-2008, Space technology items. General requirements for mitigation of near-earth space debris population Introduced 2009-01-01. Moscow.

2. B. Sommer, K. Landzettel, DEOS Deutsche Orbitale Servicing Mission: the in-flight technology demonstration of Germany's Robotic approach to service satellites, The NASA second international workshop on onorbit satellite servicing, 2012.

3. G. Henshaw, B. Kelm, DARPA Phoenix. Overview and Risk Reduction Plans, i-SAIRAS Conf., 2014.

4. On Orbit Servicing. Access mode: http://www.unoosa.org/pdf/pres/stsc2013/2013iaf-05E.pdf

5. B. Bischof, L. Kerstein, J. Starke, et al., Roger-Robotic geostationary orbit restorer, 8th ESA Workshop on Advanced Space Technologies for Robotics and Automation, 2004.

6. Japan's blasts a giant 'fishing net' into space to clear up some of the 100 MILLION pieces of junk in Earth's orbit, Associated Press and Abigail Beall For Mailonline, 2016. Retrieved from: http://www.dailymail.co.uk/sciencetech/article-4017316/Japan-launchesneeded-supplies-space-station.html

7. J. Reed, J. Busuets, and C. White, Grappling system for capturing heavy space debris, 2nd European Workshop on Active Debris Removal, 2012.

8. Internet-based testing in Analytical Mechanics, Issue 5, Momentum and Kinetic Momentum. Guidelines for Preparation for Internet-based Testing in Analytical Mechanics, Nizhny Novgorod, NNGASU, 2011.

9. E. Papadopoulos, S. Dubowsky, Coordinated Manipulator/Spacecraft Motion Control for Space Robotic Systems, Proceedings of International Conference on Robotics and Autanatim, Sacramento, CA, USA, 1991, pp. 1696-1701. 